\title{
Multi-rover testbed for teleconducted and autonomous surveillance, reconnaissance, and exploration
}

Wolfgang Fink, Mark A. Tarbell

Wolfgang Fink, Mark A. Tarbell, "Multi-rover testbed for teleconducted and autonomous surveillance, reconnaissance, and exploration," Proc. SPIE 7331, Space Exploration Technologies II, 73310B (20 May 2009); doi: 10.1117/12.819991

SPIE Event: SPIE Defense, Security, and Sensing, 2009, Orlando, Florida, United States 


\title{
Multi-Rover Testbed for Teleconducted and Autonomous Surveillance, Reconnaissance, and Exploration
}

\author{
Wolfgang Fink ${ }^{\mathrm{a}^{*}}$ and Mark A. Tarbell ${ }^{\mathrm{a}}$ \\ ${ }^{a}$ California Institute of Technology, Visual and Autonomous Exploration Systems Research \\ Laboratory, Division of Physics, Mathematics \& Astronomy, 1200 E California Blvd, \\ Mail Code 103-33, Pasadena, CA 91125, USA
}

\begin{abstract}
At Caltech's Visual and Autonomous Exploration Systems Research Laboratory (http://autonomy.caltech.edu) an outdoor multi-rover testbed has been developed that allows for near real-time interactive or automatic control from anywhere in the world via the Internet. It enables the implementation, field-testing, and validation of algorithms/software and strategies for navigation, exploration, feature extraction, anomaly detection, and target prioritization with applications in planetary exploration, security surveillance, reconnaissance of disaster areas, military reconnaissance, and delivery of lethal force such as explosives for urban warfare. Several rover platforms have been developed, enabling testing of cooperative multi-rover scenarios (e.g., inter-rover communication/coordination) and distributed exploration of operational areas.
\end{abstract}

Keywords: Tier-scalable reconnaissance, rover testbed, worldwide control, cloud computing, Internet, military reconnaissance, security surveillance, planetary exploration, urban warfare, delivery of explosives, hazardous environments, distributed exploration, human-robot interaction, astronaut training, autonomous self-commanding, autonomous telecommanding, teleoperation

\section{INTRODUCTION}

With multinational lunar and Mars exploration programs currently in progress or planned, robotic reconnaissance operations are called for in extreme environments such as space, including planetary atmospheres, surfaces, and subsurfaces, as well as in potentially hazardous or inaccessible operational areas on Earth. Future reconnaissance missions will require increasing degrees of operational autonomy, such as: (1) Automatic mapping of an operational area from different vantages (i.e., spaceborne, airborne, surface, subsurface); (2) automatic feature extraction and target/region-of-interest/anomaly identification within the mapped operational area; (3) automatic target prioritization for follow-up or close-up (in-situ) examination; and (4) subsequent automatic, targeted deployment and navigation/relocation of agents/sensors (e.g., to follow up on transient events).

The authors report on the development and implementation of both an indoor and outdoor testbed for Tier-scalable Reconnaissance - a novel paradigm in planetary exploration, originated by Fink et al. [1-7], that allows for distributed, science-driven, and less constrained reconnaissance of prime locations on Mars, the Moon, Titan, Venus, etc.

At Caltech's Visual and Autonomous Exploration Systems Research Laboratory (http://autonomy.caltech.edu) the authors have developed a multi-rover testbed, described in the following, that allows for near real-time interactive (or automatic) control from anywhere in the world via the Internet. It enables the implementation, field-testing, and validation of algorithms/software and strategies for navigation, exploration, feature extraction, anomaly detection, and target prioritization with applications in planetary exploration, security surveillance, investigation of disaster areas, military reconnaissance, and delivery of lethal force such as explosives for urban warfare. Such a robotic testbed opens up the opportunity for a wide scientific target audience (planetary geologists, hydrologists, astrobiologists, mission architects, physicists, roboticists, etc.) to develop and field-test remote planetary exploration strategies and tools, ranging from algorithms to hardware. Furthermore, it serves as a platform for training astronauts in teleoperating robotic assets on planetary bodies such as the Moon and Mars.

*e-mail: wfink@autonomy.caltech.edu; phone: +1-626-395-4587; website: http://autonomy.caltech.edu

Space Exploration Technologies II, edited by Wolfgang Fink, Proc. of SPIE Vol. 7331,

73310B · C 2009 SPIE · CCC code: 0277-786X/09/\$18 · doi: 10.1117/12.819991 


\section{IMPLEMENTATION OF A MULTI-ROVER TESTBED}

The following subsections 2.1 and 2.2 are in part cited from Fink et al., 2007 [4]:

\subsection{First Generation Indoor Miniature Testbed}

As a first step towards a testbed for tier-scalable reconnaissance, the authors have developed an indoor miniature testbed consisting of a 4' x 5' operational area with a mast-mounted camera "hovering" above, emulating the overhead perspective of an airborne platform (Fig. 1). Blocks of differing sizes, colors, albedos, and shapes were used to simulate "rocks" with a certain feature space. Fully maneuverable mini-rover units, equipped with onboard optical cameras, were autonomously commanded via a remote wireless telecommanding and control system to "interesting" science targets determined by a feature extraction and science goal prioritization software package (Automated Global Feature Analyzer $(A G F A)[8,9])$, using the overhead camera perspective (Fig. 1).
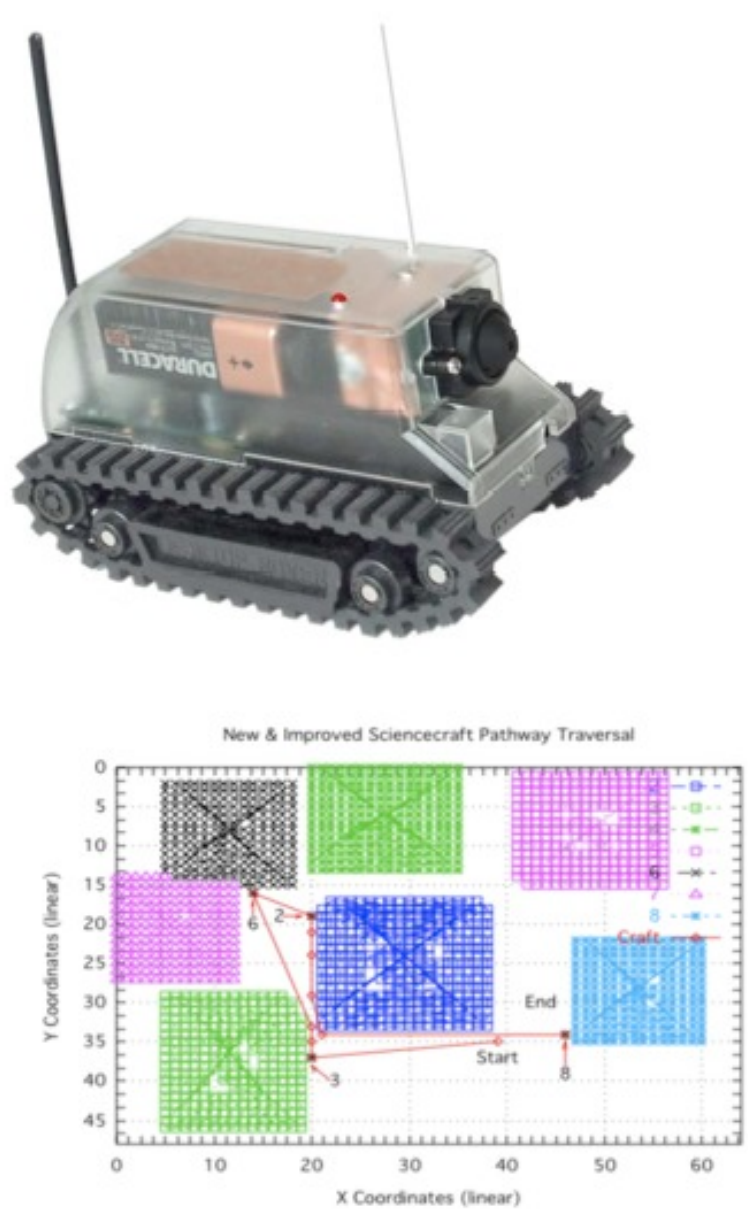

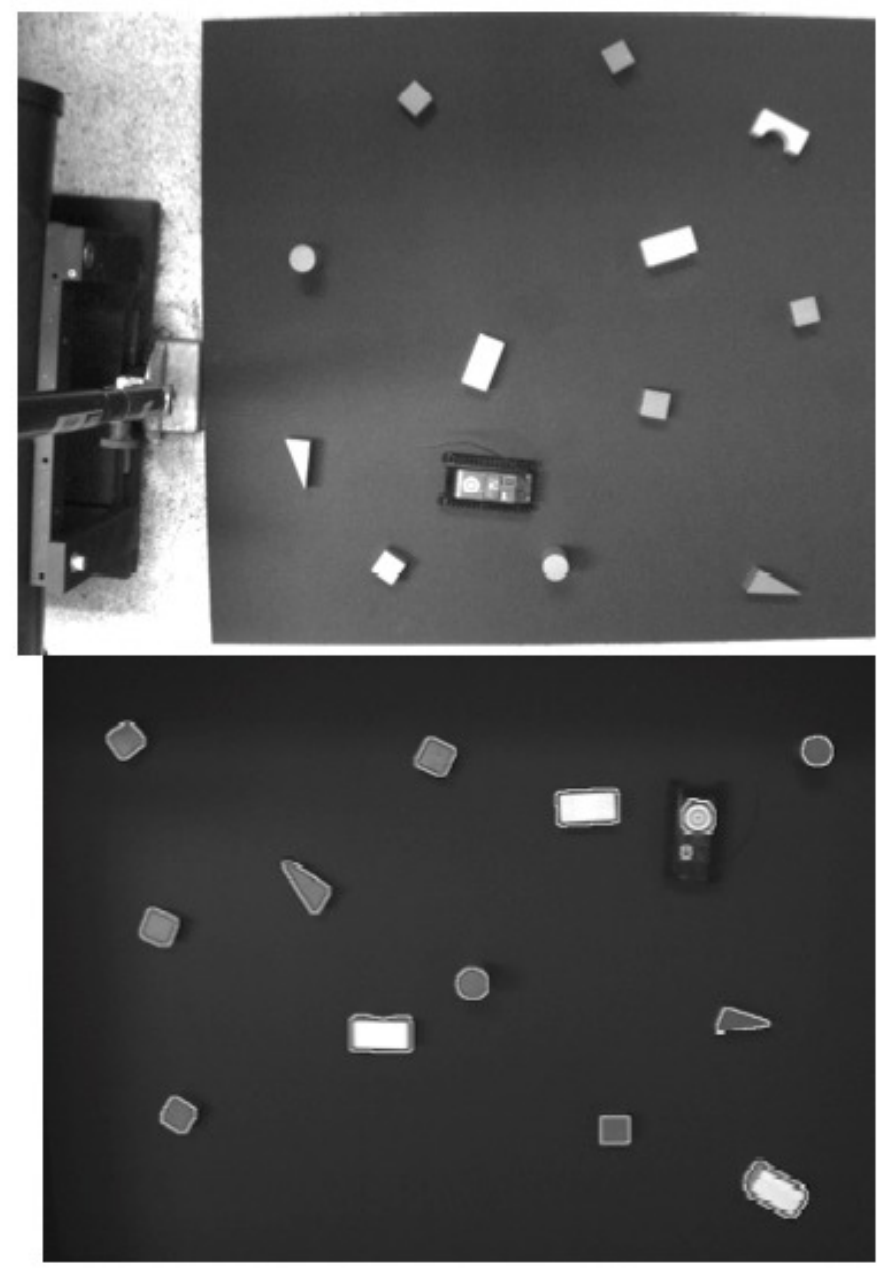

Fig. 1. Upper left: sensor-equipped (i.e., wireless onboard camera), remote controlled ground-tier agent (distributed by Plantraco). Upper right: overhead view of miniature testbed for overhead-guided autonomous surface exploration with ground-based rover unit. Lower right: image processing via the Automated Global Feature Analyzer (AGFA) [8, 9] of (science) targets within the operational area. Lower left: path planning of a collision-free ground-agent trajectory around obstacles to various science targets identified and characterized by AGFA. 
The underlying integrated software system for the miniature testbed comprised the following software components:

- Overhead image capture

- Image processing for target identification with AGFA (image segmentation and characterization, etc.)

- Overhead tracking and navigation for ground-based rovers

- Feature extraction of identified targets with AGFA

- Field site classification and science goal prioritization with AGFA

- Collision-free path planning for ground-based rovers

- Wireless commanding interface of ground-based rovers, comprising a USB interface to a wireless remote control unit.

This miniature testbed successfully proved the concept of tier-scalable reconnaissance by demonstrating closed-loop (autonomous, i.e., no human in the loop) deployments (i.e., commanding and navigation) of the mini-rover to multiple (up to seven) science targets within the operational area without collisions, exclusively guided and commanded by the overhead perspective (i.e., hovering camera).

The developed hardware and software platform allowed for:

- Testing of automated geologic field site classification and science goal prioritization algorithms (e.g., [8-11])

- Testing of navigation algorithms of ground-based science craft

- Testing of path planning algorithms

- Testing of algorithms for operating multiple science craft simultaneously within a field site for science-driven exploration in parallel

- Testing of algorithms for closed-loop (autonomous) science context driven exploration.

\subsection{Second Generation Outdoor Testbed}

To study and validate the tier-scalable reconnaissance concept under more realistic, outdoor conditions than is possible with the above miniature testbed, the authors have finished the development and implementation of an outdoor testbed (Fig. 2) consisting of remote controllable (via the Internet) robotic platforms (Fig. 3) [12, 13]. For the basic robotic hardware, WiFiBoTs [14] were utilized. The WiFiBoT's controller infrastructure is a 4G Access Cube, which serves as the central onboard processor, controlling four electric motors. A detailed description of the individual robotic ground agents and their worldwide commanding and sensor data transmission capabilities can be found in [15]. Thus, only a brief overview is given in the following.

In particular, the individual ground agents (Fig. 3) of the multi-rover testbed (Fig. 2) are equipped with:

- 4 wheel motors

- Bi-level metal chassis and sensor trays

- General-purpose, high-performance (dual-core) mini Unix workstation

- Rechargeable batteries for the wheel motors (2 hours) and for the onboard Unix workstation (4.5 hours)

- Gimbaled IP camera that is user-controllable (wireless video link)

- IEEE 1394 navigation camera with wide-angle field of view

- Two forward-looking IR proximity sensors

- Swappable sensor platform

- Real-time voice synthesizer interface

- Wireless Internet capability, i.e., TCP/IP enabled (worldwide remote control). 


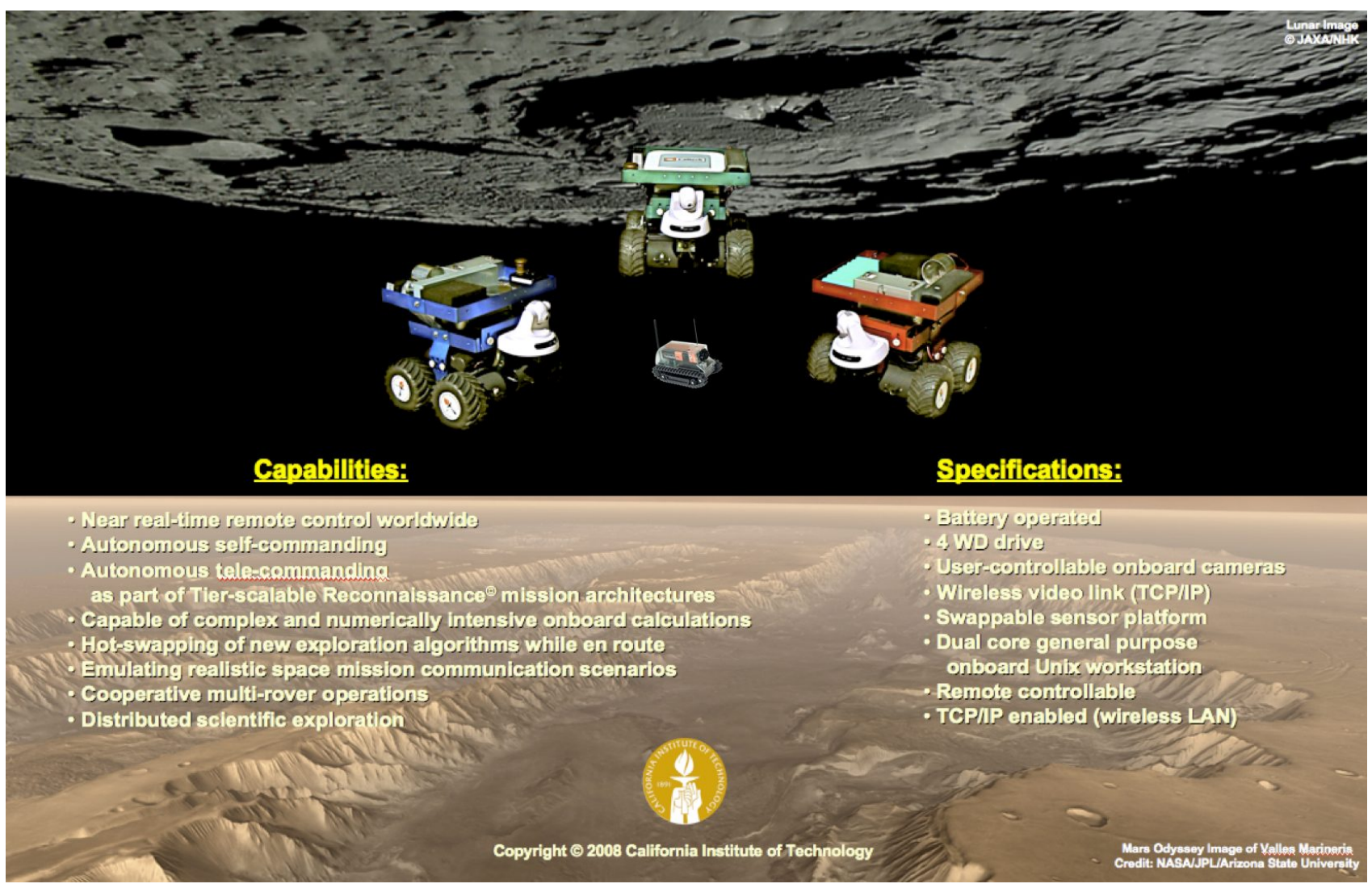

Fig. 2. Worldwide controllable (via the Internet) outdoor multi-rover testbed, representing the mobile ground-tier of the tierscalable reconnaissance mission testbed (from [13]). Center: size comparison between first generation proof-ofconcept indoor testbed agent and second generation outdoor multi-rover testbed agents.

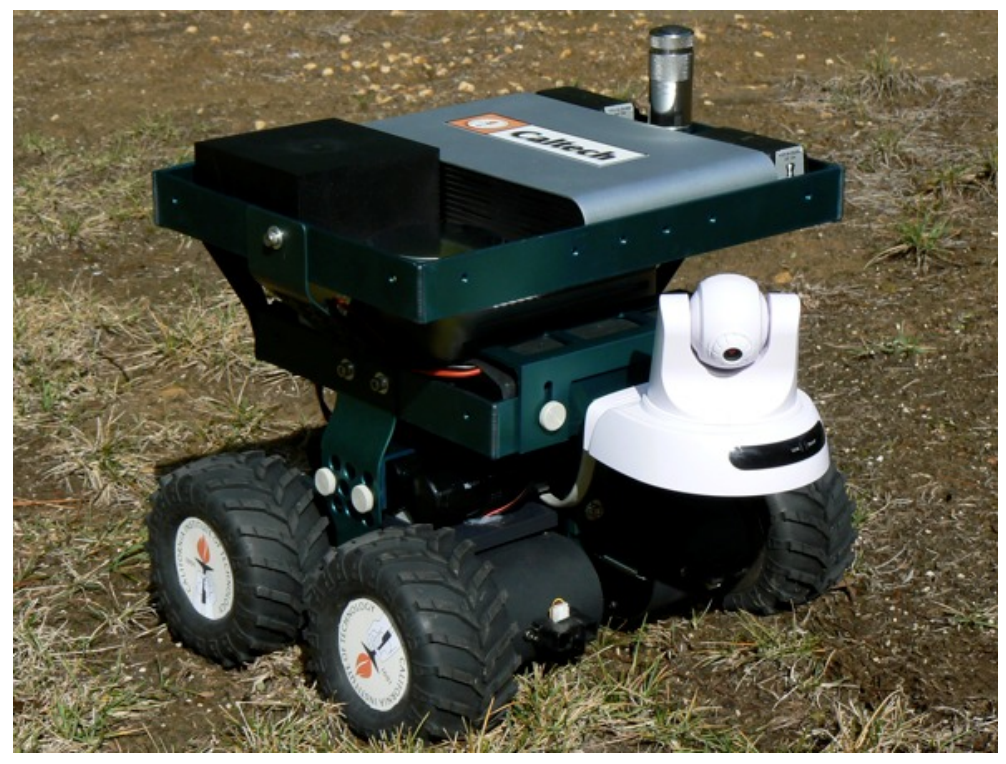

Fig. 3. Close-up of a ground-tier agent (i.e., rover), equipped with fully articulated, gimbaled digital (science) camera (white), and a navigation camera beneath (from [13]). 
To enable remote user control of the ground-tier agent, a network TCP/IP connection is first established between the CPU aboard the mobile platform (via its wireless LAN) and the computer hosting the front-end control software using a Cloud Computing concept [16, 17], wherein the mobile platform connects to one or more known "Com Servers" (Fig. 4). The Com Servers are known, established Internet entities to which both the mobile robotic platform (ground agent) and the controlling computer system connect, acting as a go-between and buffer. In this way, neither end need know the actual IP address of the other, yet an Internet connection is still established between them, with auto-reconnect in case of connection dropouts.

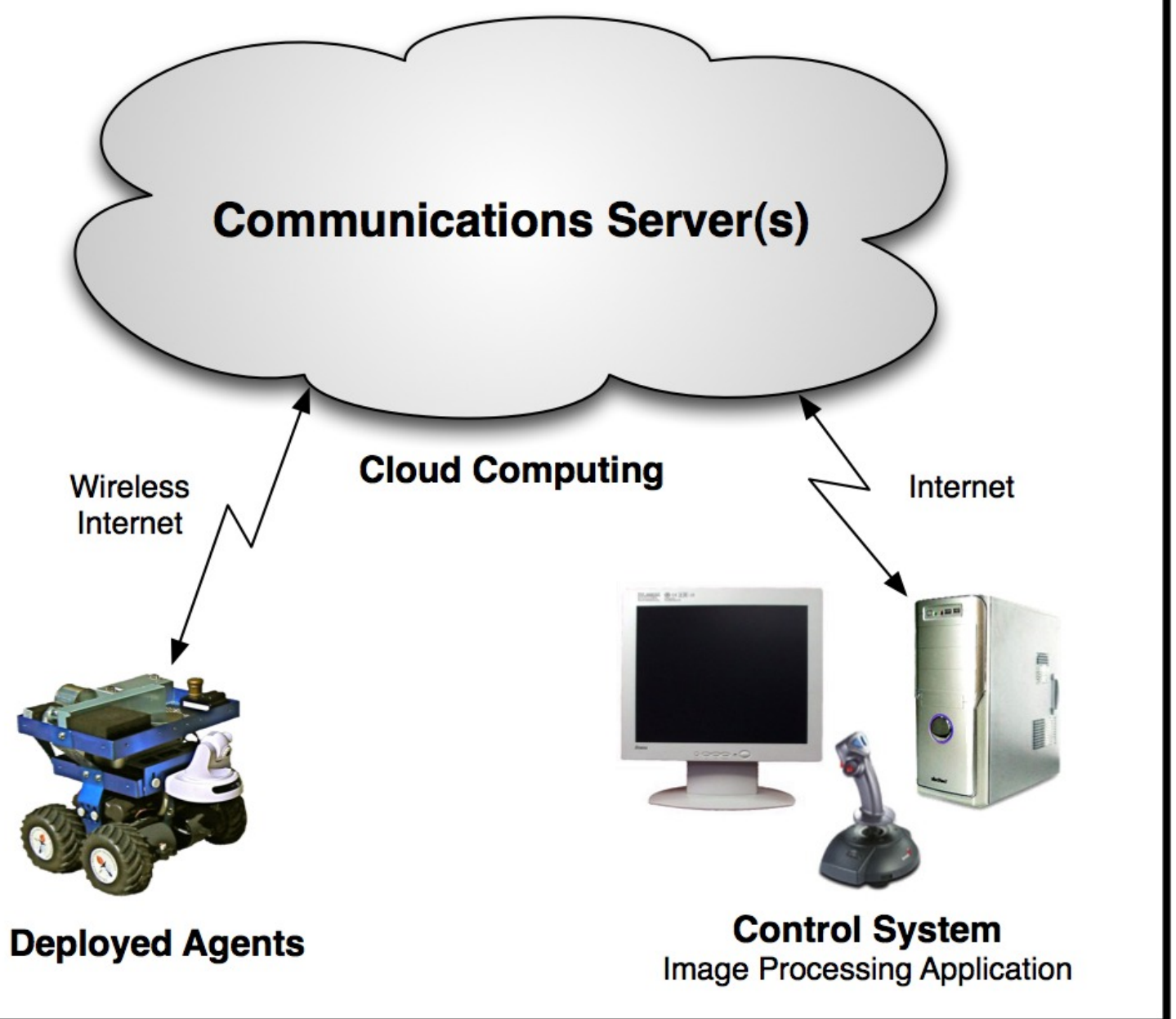

Fig. 4. Cloud Computing concept [16, 17]. Deployed agents and control system independently connect to a communication server within the cloud computing realm. In this way, agents and control systems need not search for each other; the communication server within the cloud acts as a connection go-between (see also [15]).

Once this connection is established, the ground agent is able to transmit video frames and sensor data in a packetized and compressed format. The ground agent also transmits its housekeeping data (battery level, $\mathrm{h} / \mathrm{w}$ sensor data, etc.) and awaits sensor and drive commands from the front-end software. The video and sensor data are treated similarly, however, the video data first are preprocessed into a suitable data format. This is accomplished by packetizing the video. Each non-interlaced stream frame of video data is compressed and inserted into a packet, tagging the data as to type, length, timestamp, and sequence. This has the advantage over time-division multiplexing of allowing for real-time 
synchronization to occur on the receiving end with minimal reconstruction processing. The network connection is thus used as a virtual N-receiver broadcast channel, each channel being a Q-ary data channel, providing the same general mechanism for video, sensor, or hardware housekeeping data.

For the purpose of commanding the ground-tier agent (currently interactively; later autonomously), the front-end software has an integrated video panel (Fig. 5) for displaying the transmitted video frames from the mobile platform's on-board camera (Fig. 3); it is also outfitted with a USB-based joystick device. The user's movements of the joystick are translated into camera orientation and wheel rotation commands, and are sent to the mobile platform. As the mobile platform begins to move, it also sends back video, sensor, and housekeeping data, which are displayed on the front-end. With this feedback information, a user (or automated control software for autonomous operation) is able to control the ground-tier agents interactively (or automatically) from anywhere in the world, in near real-time.

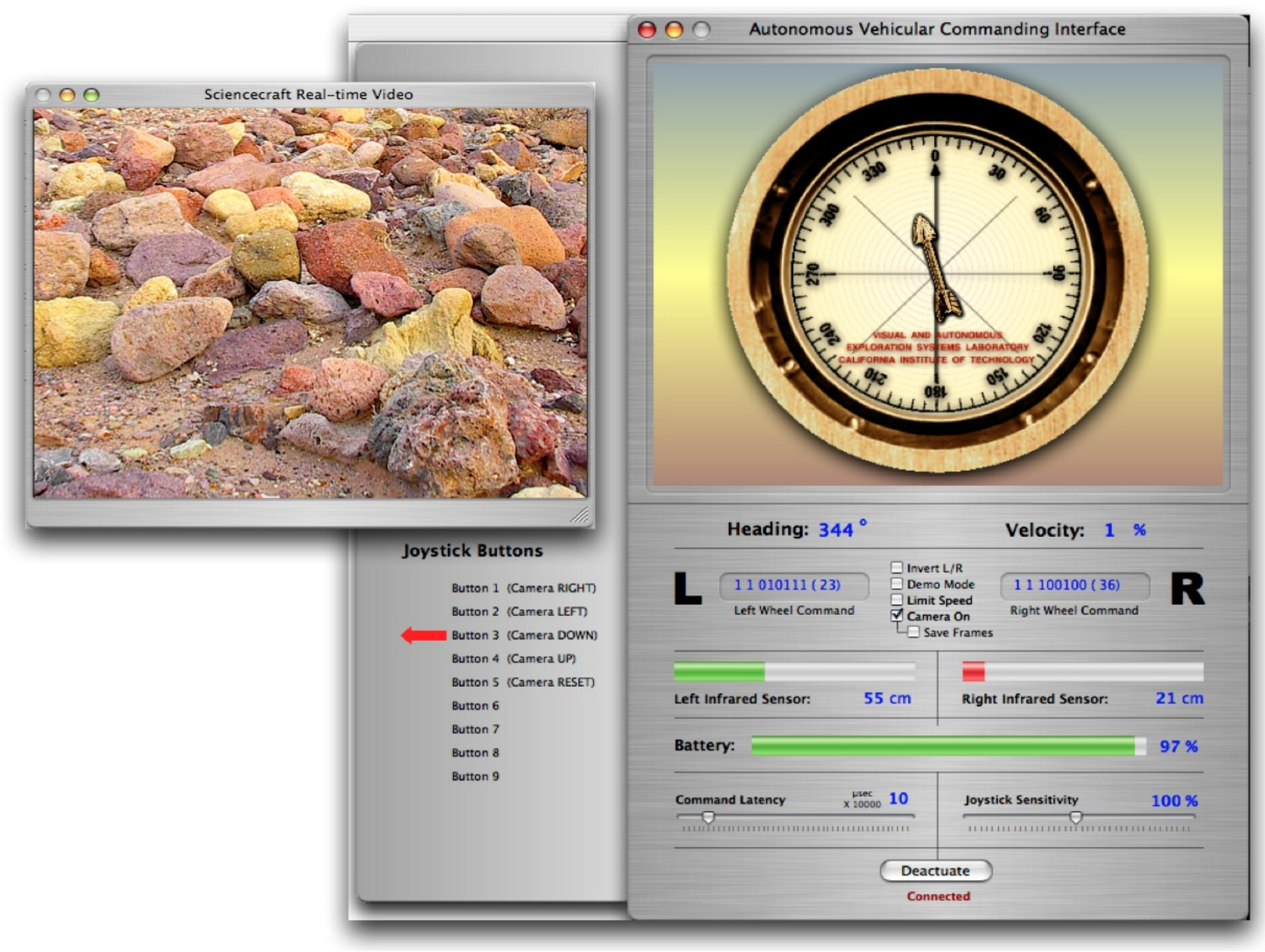

Fig. 5. Autonomous Vehicular Commanding Interface, controlling a 4WD remote robotic platform in near real-time (from $[4])$.

Figure 5 depicts the Autonomous Vehicular Commanding Interface ("software front-end") commanding the 4WD remote robotic platforms ("agents") pictured in Figs. 2 and 3. When the software front-end is networked with the agents, they are linked in a near real-time positive feedback loop: A user controlling the joystick sends drive and sensor commands to the active agent; the agent executes the commands, and replies with sensor, housekeeping, and video data sent back to the user. With this feedback information, the user (or a future automated control software for self-commanding) is able to control the ground agent(s) interactively (or automatically) from anywhere in the world, in near real-time. 
The user, controlling the joystick, is in the loop for the purpose of the development of automated software and algorithms to control the remote agents. Once developed, such automated software can be "plugged in" in lieu of the user for automatic control of the agents, with manual user override always available. Two modes of automatic control can be realized:

- Autonomous self-commanding

- Autonomous telecommanding as part of Tier-scalable Reconnaissance ${ }^{\odot}$ mission architectures $[1-7,9]$.

Representatives of expert communities and stake holders (e.g., planetary researchers, astronauts, surveillance personnel, military personnel) can interface their software packages either by remotely issuing high-level commands over the Internet, or by integrating and running their software packages locally on the onboard Unix workstation, thereby bypassing the Internet for command transmittal. In either case, it will be possible to monitor remotely the actions and camera views of the ground agents via the Autonomous Vehicular Commanding Interface (Fig. 5).

\section{DISCUSSION \& OUTLOOK}

The Caltech outdoor multi-rover testbed, presented here, comprises currently three ground agents (Figs. 2 and 3). As such it provides/enables the following capabilities $[4,5,12,13]$ :

- Near real-time interactive (or automatic) remote control worldwide

- Autonomous self-commanding

- Autonomous telecommanding as part of Tier-scalable Reconnaissance ${ }^{\odot}$ mission architectures

- Capable of complex and numerically intensive onboard calculations

- Hot-swapping of new exploration algorithms while en route

- Emulating realistic (space) mission communication scenarios (e.g., by introducing precise communication/command execution latencies)

- Implementation, field-testing, and validation of algorithms/software and strategies for navigation, exploration, feature extraction and anomaly detection (e.g., $[8,9])$, as well as target/science goal prioritization (e.g., $[8,10$, 11])

- Cooperative multi-rover operations, e.g., inter-rover communication and coordination to navigate and explore operational areas (either teleconducted or autonomously)

- Field-testing of (novel) instrument and sensor equipment

- Distributed (scientific) exploration, surveillance, reconnaissance of operational areas, and payload delivery (e.g., in-situ sensors or explosives).

There are numerous applications for such a multi-rover testbed. For example, in the case of the Moon, the telecommanded or autonomous exploration of craters devoid of sunlight and the exploration of the far side of the Moon are of vital importance to determine whether there are permanent water ice deposits for the potential establishment of a human base on the Moon. Similarly, on Mars, autonomous robotic exploration of high-risk high-yield sites is among the high priority goals of future planetary exploration. Moreover, for both the Moon and Mars local teleoperations of robots (i.e., astronaut-robot interactions) are critical for the purpose of resource exploitation and transport of supplies in support of a temporary or permanent human presence. The multi-rover testbed serves as a software and hardware test platform for autonomous operations as well as for training astronauts in teleoperating rovers.

In addition to applications in planetary exploration, the multi-rover testbed presented here and its agents (i.e., rovers) can be employed in security surveillance, reconnaissance of disaster areas, military reconnaissance, and delivery of lethal force such as explosives for urban warfare.

It is important to point out that a hidden value of the above multi-rover testbed lies in the software infrastructure (i.e., Cloud Computing (Fig. 4) coupled with the Autonomous Vehicular Commanding Interface (Fig. 5)). This was developed 
to control the agents from anywhere in the world and to transmit data from the rovers back to the commanding post. As such, this software infrastructure is independent from the actual physical embodiment of the mobile agents, and is directly transplantable into other agents (e.g., larger rovers, maritime vessels, aerial platforms, etc.). The single area subject to modification, and specific to the respective agent embodiment used, is the actual interface to the physical motors and steering mechanisms of the actual agents deployed. The Autonomous Vehicular Commanding Interface and the Cloud Computing infrastructure are generic and reusable.

\section{ACKNOWLEDGEMENTS}

The work described in this publication was carried out at the California Institute of Technology under partial support of the National Science Foundation Grant EEC-0310723.

\section{REFERENCES}

1. Fink W, Dohm JM, Tarbell MA, Hare TM, Baker VR (2005) Next-Generation Robotic Planetary Reconnaissance Missions: A Paradigm Shift; Planetary and Space Science, 53, 1419-1426.

2. Fink W, Dohm JM, Tarbell MA, Hare TM, Baker VR, Schulze-Makuch D, Furfaro R, Fairén AG, Ferré TPA, Miyamoto H, Komatsu G, Mahaney WC (2006) Autonomous Tier-Scalable Reconnaissance Missions For Remote Planetary Exploration; Proceedings of the 4th International Planetary Probe Workshop 2006, Pasadena.

3. Fink W, Dohm JM, Tarbell MA, Hare TM, Baker VR, Schulze-Makuch D, Furfaro R, Fairen AG, Ferre TPA, Miyamoto H, Komatsu G, Mahaney WC (2007) Tier-Scalable Reconnaissance Missions For The Autonomous Exploration Of Planetary Bodies; IEEE Aerospace Conference Proceedings, paper \#1199; DOI: 10.1109/AERO.2007.352715.

4. Fink W, George T, Tarbell MA (2007) Tier-Scalable Reconnaissance: The Challenge of Sensor Optimization, Sensor Deployment, Sensor Fusion, and Sensor Interoperability; Proc. SPIE, Vol. 6556, 655611 (2007); DOI:10.1117/12.721486 (invited paper).

5. Fink W, Tarbell MA, Jobling FM (2008) Tier-Scalable Reconnaissance - A Paradigm Shift in Autonomous Remote Planetary Exploration of Mars and Beyond; Chapter 1 in "Planet Mars Research Focus", Ed. L. A. Costas. Nova Science Publishers, Hauppauge, NY. ISBN: 1-60021-826-1.

6. Fink W, Tarbell MA (2007) Tier-Scalable Reconnaissance $\bigodot$ - A Paradigm Shift in Autonomous Robotic Planetary Exploration; 7th IAA International Conference on Low-Cost Planetary Missions 2007, Pasadena, CA, abstract and poster.

7. Caltech's Visual and Autonomous Exploration Systems Research Laboratory Web site for Tier-Scalable Reconnaissance (by W. Fink) http://autonomy.caltech.edu/autonomy/tierscalable.html

8. Fink W, Datta A, Dohm JM, Tarbell MA, Jobling FM, Furfaro R, Kargel JS, Schulze-Makuch D, Baker VR (2008) Automated Global Feature Analyzer (AGFA) - A Driver for Tier-Scalable Reconnaissance; IEEE Aerospace Conference Proceedings, paper \#1273; DOI: 10.1109/AERO.2008.4526422.

9. Fink W, Datta A, Dohm JM, Tarbell MA, Jobling FM, Furfaro R, Kargel JS, Schulze-Makuch D, Lunine JI, Baker VR (2008) Automated Global Feature Analyzer (AGFA) for the Intelligent and Autonomous Robotic Exploration of the Solar System [abstract 1883]. In 39th Lunar and Planetary Science Conference Abstracts [CD-ROM], Lunar and Planetary Institute, Houston.

10. Fink W (2006) Generic Prioritization Framework for Target Selection and Instrument Usage for Reconnaissance Mission Autonomy, Proceedings of IEEE World Congress on Computational Intelligence (WCCI) 2006, Vancouver, Canada, 11116-11119.

11. Furfaro R, Dohm JM, Fink W, Kargel JS, Schulze-Makuch D, Fairén AG, Ferré PT, Palmero-Rodriguez A, Baker VR, Hare TM, Tarbell M, Miyamoto HH, Komatsu G (2007) The Search for Life Beyond Earth Through Fuzzy Expert Systems; Planetary and Space Science, 56, 448-472.

12. Fink W and Tarbell MA (2007) Tier-scalable Reconnaissance Mission Test Bed: Implementation of Ground-Tier [abstract 2410]. In 38th Lunar and Planetary Science Conference Abstracts [CD-ROM], Lunar and Planetary Institute, Houston. 
13. Fink W and Tarbell MA (2008) Multi-Rover Test Bed for Tele-Conducted and Autonomous Surface Operations for the Moon and Mars [abstract 2472]. In 39th Lunar and Planetary Science Conference Abstracts [CD-ROM], Lunar and Planetary Institute, Houston.

14. Robosoft, http://www.robosoft.fr/eng/

15. Fink W and Tarbell MA (2009) CYCLOPS: A Mobile Robotic Platform for Testing and Validating Image Processing and Autonomous Navigation Algorithms in Support of Artificial Vision Prostheses; submitted to Computer Methods and Programs in Biomedicine.

16. Chellappa R (1997) Cloud Computing - Emerging Paradigm for Computing. INFORMS 1997, Dallas, TX.

17. Hayes B (2008) Cloud Computing. Communications of the ACM, Volume 51, Number 7. 